LA-UR-94-3563 (Final)

Title:

Author(s):

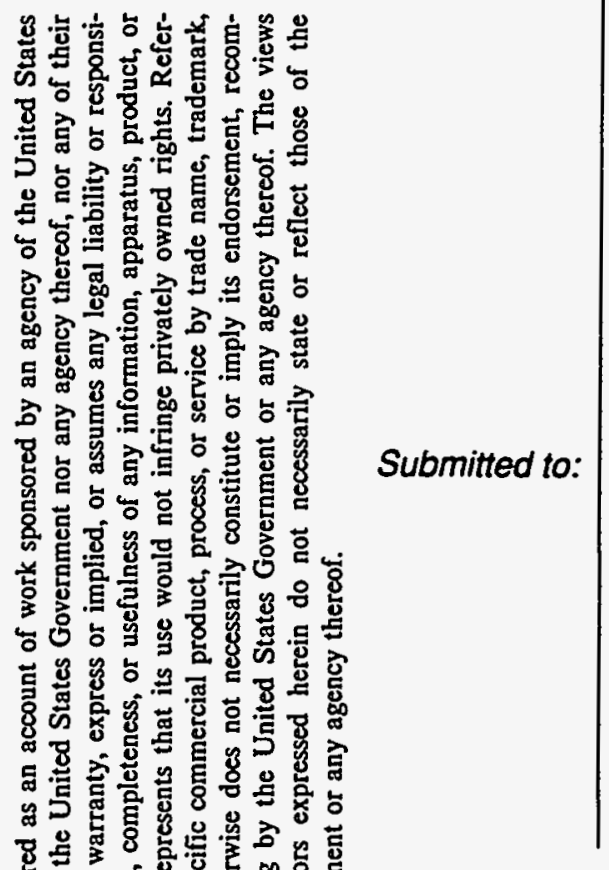

J. D. Bowman, C. M. Frankle, A. A. Green, J. N. Knudson, S. I. Penttilä, S. J. Seestrom, Y.-F. Yen, V. W. Yuan, B. E. Crawford, N. R. Roberson, C. R. Gould, D. G. Haase, L. Y. Lowie, G. E. Mitchell, S. I. Stephenson, P. P. J. Delheij, E. I. Sharapov, H. Postma, Y. Masuda, M. linuma, A. Masaike, Y. Matsuda, and K. Fukuda
First International Symposium on Symmetries
in Subatomic Physics
Taiwan, Republic of China, May 16-18, 1994

and

\section{Conference on the Intersections Between} Particle and Nuclear Physics St. Petersburg, Florida, May 31-June 6, 1994

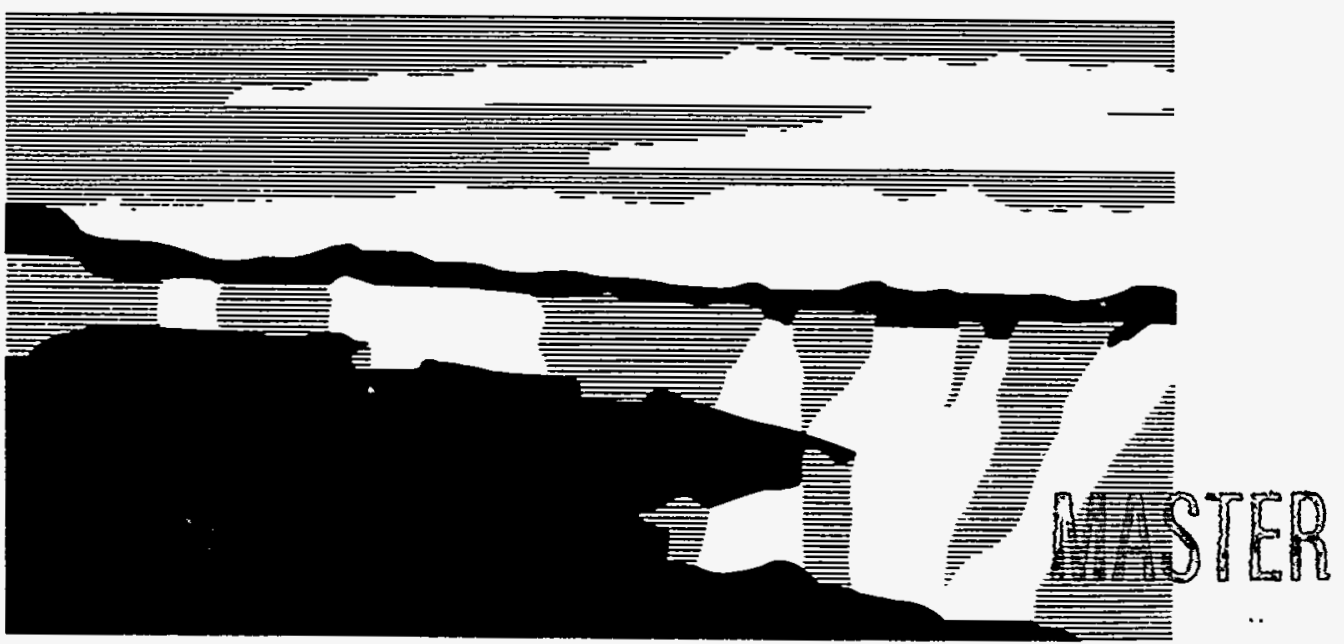

Los Alamos National Laboratory, an affirmative action/equal opportunity empldyer, is operated by the University of California tor the U.S. Department of Energy under contract W-7405-ENG-36. By acceptance of this article, the publisher recognizes that the U.S. Government retains a nonexclusive, royahy-free license to publish or reproduce the published form of this contribution, or to allow others to do so, for U.S. Government purposes. The Los Alamos National Laboratory requests that the publisher identify this anticle as work performed under the auspices of the U.S. Department of Energy. 


\section{DISCLAIMER}

\section{Portions of this document may be illegible in electronic image products. Images are produced from the best available original document.}




\title{
PARITY VIOLATION IN THE COMPOUND NUCLEUS
}

J. D. Bowman, C. M. Frankle, A. A. Green, J. N. Knudson, S. I. Penttilä, S. J. Seestrom, Yi-Fen Yen, and V. W. Yuan

Los Alamos National Laboratory, Los Alamos NM 87545, USA

B. E. Crawford and N. R. Roberson

Duke University, Durham NC 27706 and TUNL, USA

C. R. Gould, D. G. Haase, L. Y. Lowie, G. E. Mitchell, and S. I. Stephenson North Carolina State University, Raleigh NC 27695 and TUNL, USA

$$
\text { P. P. J. Delheij }
$$

TRIUMF, 4004 Wesbrook Mall, Vancouver, BC, Canada VT6 2A3

E. I. Sharapov

Joint Institute for Nuclear Research, 141980 Dubna, Russia

$$
\text { H. Postma }
$$

University of Technology, P. O. Box 5046, 2600 GA, Delft, The Netherlands

Y. Masuda and H. M. Shimizu

National Laboratory for High Energy Physics, 1-1 Oho, Tsukuba-shi 305, Japan

M. Iinuma, A. Masaike, and Y. Matsuda

Department of Physics, Kyoto University, Kyoto 606, Japan

\author{
K. Fukuda \\ Department of Physiology, Meiji College of Oriental Medicine, \\ Hiyoshi-cho, Funai-gun, Kyoto 629, Japan \\ (The TRIPLE Collaboration)
}

\begin{abstract}
The status of parity violation in the compound nucleus is reviewed. The results of previous experimental results obtained by scattering polarized epithermal neutrons from heavy nuclei in the 3-p and 4-p p-wave strength function peaks are presented. Experimental techniques are presented. The
\end{abstract}


extraction of the mean squared matrix element of the parity-violating interaction, $M^{2}$, between compound-nuclear levels and the relationship of $M^{2}$ to the coupling strengths in the meson exchange weak nucleon-nucleon potential are discussed. The tendency of measured asymmetries to have a common sign and theoretical implications are discussed. New experimental results are presented that show that the common sign phenomenon is not universal, as theoretical models developed up to now would predict.

This talk is dedicated to our friend and colleague Professor Ernest M. Henley, who has done so much to inspire the understanding and study of fundamental symmetries in the nucleus.

\section{Introduction}

I will review the study of the parity-violating interaction in compound nuclei and present some new results on the tendency of parity-violating asymmetries measured in ${ }^{232} \mathrm{Th}$ to have a common sign.

A compound nucleus (CN) is formed when a nucleus $A$ captures a neutron to form an excited state of the $A+1$ daughter nucleus. The excitation energy of $\mathrm{CN}$ states in the daughter is typically $5 \mathrm{MeV}$ in a heavy nucleus. The corresponding level spacing, $D$, is of the order of a few eV. If the wave function of a $\mathrm{CN}$ level is expanded on a singleparticle basis, the number of states involved will be of order $\Gamma / D \sim 10^{5}$. It is impossible to calculate the wave function of a $\mathrm{CN}$ level since uncertainties in the nuclear Hamiltonian would lead to qualitative changes in the size of expansion coefficients. The statistical model of the $\mathrm{CN}$ assumes that the expansion coefficients of the wave function, as well as matrix elements, behave like independent Gaussian random variables. This model has been quite successful in describing features of the $\mathrm{CN}$ such as level-spacing distributions and average neutron and $\gamma$ decay rates.

It seems paradoxical that the compound nucleus could play any useful role in the study of discrete symmetries such as parity violation (PV) or time reversal. In this talk I will show (1) that the scattering of epithermal (1-1000 eV) through $\mathrm{CN}$ levels shows remarkably large PV asymmetries, (2) that the magnitude of these asymmetries can be qualitatively understood and quantitatively related to the coupling strengths of the weak meson exchange potential, and (3) that measured asymmetries exhibit unexpected nonstatistical behavior.

\section{The Experiment}

The parity-violating longitudinal asymmetry for the $\mathrm{i}^{\text {th }} p$-wave resonance is $P_{i}=$ $\left(\sigma_{+}-\sigma_{-}\right) /\left(\sigma_{+}+\sigma_{-}\right)$, where $\sigma_{+}$and $\sigma_{-}$are the resonance cross sections for positive and negative helicity neutrons. The TRIPLE collaboration has developed an apparatus to take advantage of the very high neutron fluxes available at LANSCE to measure several parity-violating asymmetries in a single target. The apparatus is described in detail in Roberson et al. ${ }^{1}$ The $500-\mu$ sec-wide $800-\mathrm{MeV}$ pulses from the LAMPF LINAC are 
injected into the Proton Storage Ring at the rate of $20 \mathrm{~Hz}$ and time compressed into triangular pulses $250 \mathrm{nsec}$ wide at the base. These pulses impinge on a tungsten target surrounded by a water moderator. The intensity of the epithermal neutron flux at neutron energy $E$ is approximately given by $d N / d E=0.01(1 / E) f \Omega(I / e)$, where $I$ is the proton current (typically $70 \mu \mathrm{A}$ ), $e$ is the quantum of charge, $\Omega$ is the solid angle, and $f$ is the fraction of the $13-\mathrm{cm}$ by $13-\mathrm{cm}$ moderator viewed by the collimator system. It is the large neutron intensity that makes the measurements described here possible.

The apparatus developed by the TRIPLE collaboration is designed to make simultaneous measurements of the longitudinal asymmetries of several $p$-wave resonances. The apparatus is shown schematically in Fig. 1 . First, the neutrons pass through a flux monitor consisting of a pair of ionization chambers; the first contains ${ }^{3} \mathrm{He}$ and the second ${ }^{4} \mathrm{He}$ at atmospheric pressure. The ${ }^{3} \mathrm{He}$ ion chamber responds to both neutrons and photons, while the ${ }^{4} \mathrm{He}$ ion chamber responds only to photons. The signals from each of these ion chambers are amplified by operational amplifiers and digitized by voltage-to-frequency converters. The neutron flux is measured for each burst (with $0.1 \%$ accuracy) by subtracting the digitized ${ }^{4} \mathrm{He}$ signal from the digitized ${ }^{3} \mathrm{He}$ signal.

The neutrons next pass through a polarized-proton spin filter. ${ }^{2}$ The protons in a sample of radiation damaged $\mathrm{NH}_{4}$ are dynamically polarized by the combination of

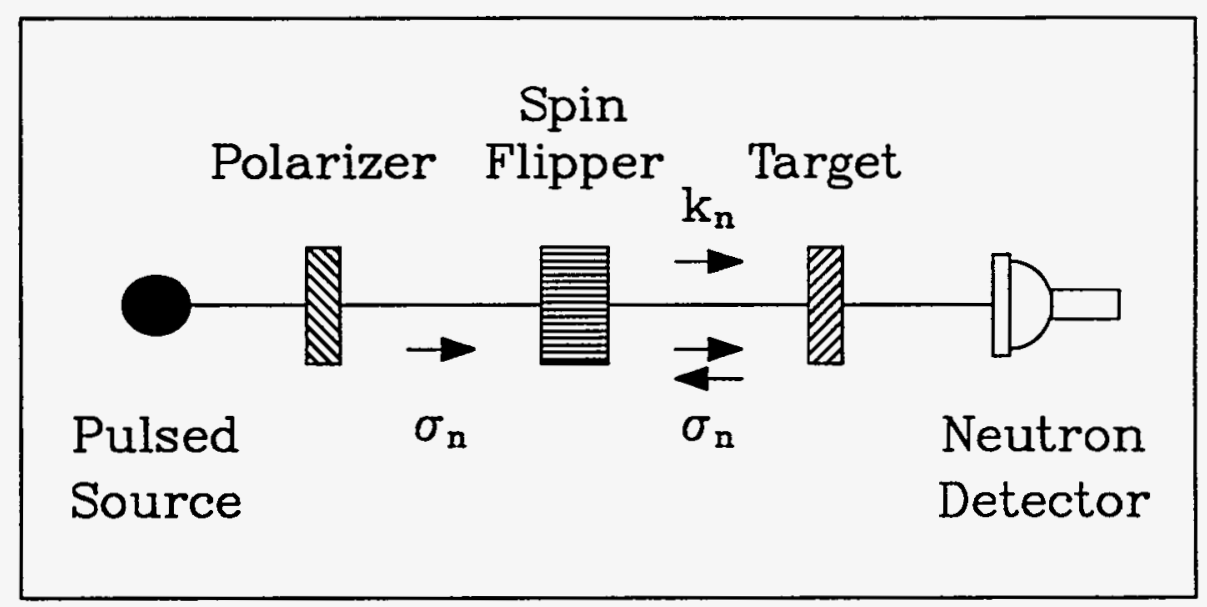

Fig. 1. Schematic view of the apparatus. Neutrons from the pulsed source pass through a polarizing filter, a cell of longitudinally polarized protons, where one helicity state is filtered out leaving a beam of longitudinally polarized neutrons. The neutron spin is reversed or not reversed by passing through a system of magnetic fields, the spin flipper. After passing through the target, the neutrons are detected by a neutron detector. The neutron energy is measured by the time of flight between the pulsed source and the detector. 
cooling to $1.1 \mathrm{~K}$, a 5.0 -T longitudinal magnetic field, and microwave pumping. The cross section for neutron spin parallel to the proton spin is much smaller than for neutron spin anti-parallel. The anti-parallel neutron spin is filtered out, producing a polarized neutron beam for neutron energies between $0.1 \mathrm{eV}$ and $50 \mathrm{keV}$. We achieved neutron beam polarization of $70 \%$.

In order to study many resonances in the same nucleus simultaneously, it is necessary to be able to reverse the neutron polarization over a wide range of neutron energies. This is accomplished by a "spin flipper," a system of magnetic fields located after the polarizer. The spin flipper has a field along the beam direction that blends into the stray field of the superconducting solenoid of the polarizer. This 0.01-T longitudinal field smoothly decreases, changes direction at the spin-flipper midpoint, and increases to be opposite and equal to the initial field. A longitudinally polarized neutron near the spinflipper symmetry axis experiences no torque and passes through the spin flipper with its spin direction unchanged. In order to reverse neutron spin direction, a transverse field is applied. This field smoothly builds up and decreases over the 1-meter length where the longitudinal field reverses direction. The moving neutron experiences a field that is initially parallel to its velocity and then rotates to be anti-parallel over a distance of 1 meter. If the Larmor frequency is large compared to the rate at which the field direction rotates, the neutron spin follows the field direction and is reversed. The neutron spin direction is controlled by turning the transverse field on or off. Calculations reported in Roberson et $a l .{ }^{1}$ show that the neutron spin is efficiently reversed for neutron energies between 0.1 and $1000 \mathrm{eV}$. Thick samples, $n \sigma$ from 1 to 3 , are placed in the downstream part of the spin-flipper solenoid. The targets were cooled to liquid nitrogen temperature to reduce Doppler broadening.

The neutron detector is located 56 meters from the spallation source. It consists of an array of $55^{10} \mathrm{~B}$ loaded liquid scintillator cells arranged in a hexagonal pattern. The neutrons are detected by the reaction $n+{ }^{10} \mathrm{~B} \rightarrow{ }^{7} \mathrm{Li}^{*}+{ }^{4} \mathrm{He}+Q_{n}$, followed by ${ }^{7} \mathrm{Li}^{*} \rightarrow$ ${ }^{7} \mathrm{Li}+478 \mathrm{KeV} \gamma$. The neutrons first thermalize by elastic collisions with the hydrogen nuclei in the mineral oil base of the scintillator and then are captured by the ${ }^{10} \mathrm{~B}$. The detector is $90 \%$ efficient up to neutron energies of $10 \mathrm{KeV}$. This detector is described has been described Yen et al. ${ }^{3}$

The data acquisition system operated in a hybrid digital-current mode. The 55 signals from photo-multiplier tubes viewing the scintillator cells were discriminated and the digital pulses sent to the data acquisition system located 150 meters away. The digital signals were shaped and added together and the analog sum was converted to digital signals by a transient digitizer. This unit periodically sampled the combined anode signals and encoded the digitized signal into a 12-bit word. The resulting sequences of digitized signals were added into a 8192-channel summation memory located in CAMAC. The memory was read out by the data acquisition computer after every 200 beam pulses were accumulated. The combined rate of the 55 detectors was as large as $500 \mathrm{MHz}$. The advantages of the hybrid technique are: (1) The ability to standardize the signal pulses 
before recording; this is particularly important in situations such as the present one where different reaction paths lead to widely varying detector responses. (2) The dead time is small. The dead time was of order $20 \%$, the rate in a single detector times the dead time in the signal processing time for that detector, $20 \mathrm{nsec}$. (3) Low cost. The information in the 55 signals is recorded without loss of statistical accuracy in one rather than 55 channels of data acquisition hardware. (4) Small data transfer time. It is necessary to transfer the data from the digitizer memory to a summation memory between beam bursts, $20 \mathrm{msec}$. With the hybrid technique one, not all 55 memory registers need to be transferred.

Several techniques were used to reduce noise and systematic errors. One sixtieth of a second after the data from each beam pulse were encoded by the transient digitizer, the data from a second beam-off time interval were encoded and subtracted from the summation memory. This procedure reduced the size of noise signals with the $60-\mathrm{Hz}$ period of the line frequency by two orders of magnitude. The spin was reversed every ten seconds in the eight-step spin flipper sequence RNNRNRRN, which eliminates drifts up to second order. For no reversal, $N$, the spin-flipper transverse field was off. For reversal, $R$, the transverse field direction was alternated so that, on the average, the stray transverse field was zero. This alternation of the transverse field direction eliminates the effects of the stray transverse field in first order. The spin direction was independently reversed by reversal of the polarization direction of the protons in the spin filter. This was done by tuning the microwave pumping frequency to select different transitions. The combination of spin-flipper and spin-filter reversal eliminates any systematic errors from either reversal method in first order. The leading systematic effect from the combined reversal method is the product of the first-order effects in both. The first-order effect from the spin-flipper reversal is estimated to be less than $10^{-10}$ and the systematic effect from changing the microwave pumping frequency is thought to be zero. Systematic errors were shown to be less than a few $10^{-5}$ from in situ measurements of $s$-wave impurity resonances, while measured longitudinal asymmetries were typically $10^{-3}$ to $10^{-1}$. Thus, we believe systematic errors to be negligible compared to other errors or the size of nonzero asymmetries in the results reported below.

\section{Interpretation of the Data}

The PV asymmetry for the $\mathrm{i}^{\text {th }}$ level, $P_{i}$, may be written as a perturbation series. ${ }^{4-6}$

$$
P_{i}=2 \sum_{j} \frac{V_{i j}}{E_{i}-E_{j}} \frac{g_{j}}{g_{i}},
$$

where $E_{i}$ and $E_{j}$ are the energies of the $p$-wave resonance and the admixed $s$-wave resonances, $V_{i j}$ is the matrix element of the PV interaction between states $i$ and $j$, and $g_{i}^{2}$ and $g_{j}^{2}$ are their neutron widths. The energies and neutron widths are known experimen- 
tally. According to the statistical model of the $\mathrm{CN}$, the neutron-decay amplitudes $g_{i}$ and $g_{j}$ and the matrix elements $V_{i j}$ are mean-zero Gaussian random variables. It is to be expected that measured asymmetries will show large fluctuations due to the statistical character of $V_{i j}, E_{i}-E_{j}, g_{i}$ and $g_{j}$. Since each of these quantities can be either positive or negative, it was generally believed that $\mathrm{PV}$ asymmetries would have random signs. The 1991 measurements of the TRIPLE collaboration on ${ }^{232} \mathrm{Th}^{11}$ brought this expectation into question. Seven out of seven measured asymmetries with greater than 2.5 standard deviation effects had positive asymmetries. In addition, the $63.5-\mathrm{eV}$ resonance in the nearby nucleus ${ }^{238} \mathrm{U}$ showed a positive asymmetry. ${ }^{10}$

Figure 2 summarizes the available data on PV asymmetries. The data are divided into two groups corresponding to the 3-P and 4-P $p$-wave strength function peaks. The asymmetries have been normalized so that data from all nuclei can be directly compared. On average, $g_{j} / g_{i} \sim 1 / \sqrt{E}, V_{i j} \sim \sqrt{d}$, and $E_{i}-E_{j} \sim d$, where $E$ is the energy of the $p$ wave resonance and $d$ is the $s$-wave level spacing. Therefore, in order to compare data

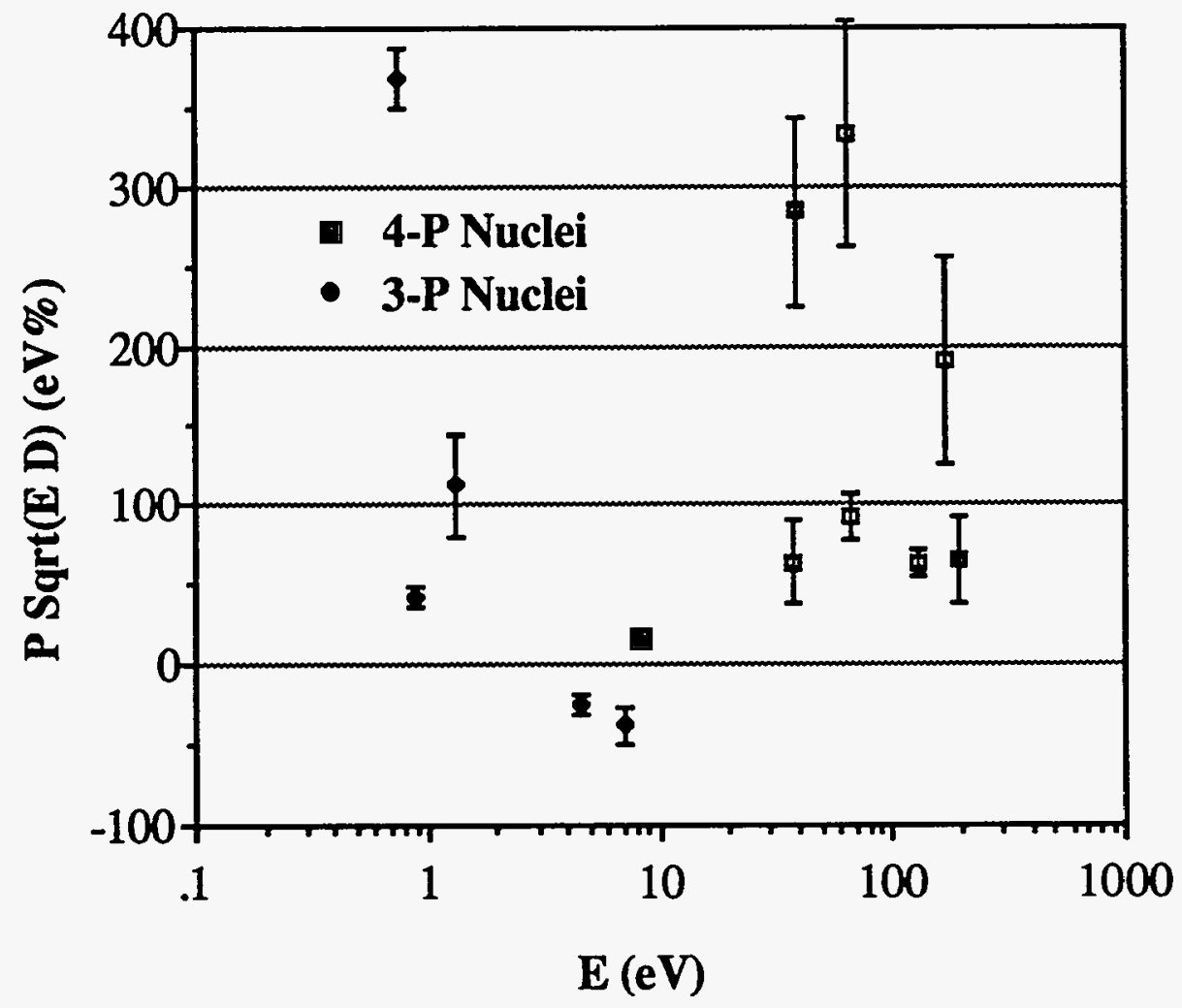

Fig. 2. Summary of available data on parity-violating asymmetries that differ from zero by more than 2.5 times their experimental error. ${ }^{7-13}$ The asymmetries have been normalized so that data from all nuclei can be directly compared. See text. 
for different resonance energies and from different nuclei, the measured asymmetries have been multiplied by $\sqrt{E d}$. In addition, the 3-P asymmetries have been multiplied by $\pi / 2$ to account for channel spin mixing, which is present in the 3-P nuclei but not in the 4-P nuclei. For the ${ }^{232}$ Th data, $P \sqrt{E d}=145 \pm 51 \% \mathrm{eV}$ or $P \sqrt{E}=36 \pm 12 \% \sqrt{\mathrm{eV}}$. For the single greater-than-2.5- standard-deviation result in ${ }^{238} \mathrm{U}, P \sqrt{E d}=92 \mathrm{eV} \%$. For the 3-P nuclei, $P \sqrt{E d}=117 \pm 72 \mathrm{eV} \%$. The errors are calculated from the fluctuations in the measured asymmetries, not from the experimental errors. There is strong evidence that the asymmetries for ${ }^{232} \mathrm{Th}$ are not random and have a non-zero average. The one non-zero asymmetry for ${ }^{238} \mathrm{U}$ has the same sign as the ${ }^{232} \mathrm{Th}$ asymmetries and a value consistent with the average ${ }^{232} \mathrm{Th}$ asymmetry. However, the average of the 3-P asymmetries is consistent with both zero and the non-zero average of the ${ }^{232} \mathrm{Th}$ asymmetries. We will discuss the interpretation of the large average asymmetry for ${ }^{232} \mathrm{Th}$ below.

As expected, the measured asymmetries have large fluctuations. The first experiment that observed more than one non-zero asymmetry in a single nucleus, ${ }^{238} \mathrm{U}$, was reported by the TRIPLE collaboration in $1990 .{ }^{10}$ This paper introduced a method, based on the statistical model of the $\mathrm{CN}$ and using likelihood analysis, for extracting $M$, the root-mean-squared matrix element of the PV interaction between $\mathrm{CN}$ states, from experimental asymmetry data in a model-independent way. We consider the analysis of a set of measurements of PV asymmetries of CN states. Equation (1) can be rewritten as:

$$
P_{i}=\sum_{j} A_{i j} V_{i j},
$$

where $A_{i j}=\left(2 / E_{i}-E_{j}\right)\left(g_{i} / g_{j}\right)$. The squares of the $A_{i j}$ are known since $E_{i}$ and $E_{j}$ and the neutron widths of the states, $g_{i}^{2}$ and $g_{j}^{2}$, are known experimentally. We assume that the $V_{i j}$ are independent Gaussian random variables with a common variance, $M^{2}$, and mean zero. $M^{2}$ is the mean-squared matrix element of the PV interaction between nuclear states. The quantity, $Q_{i}=P_{i} / B_{i}$, where $B_{i}^{2}=\sum_{j} A_{i j}^{2}$, can be written as:

$$
Q_{i}=\frac{1}{B_{i}} \sum_{j} A_{i j} V_{i j} \quad \text { and } \quad \sum_{j}\left(\frac{A_{i j}}{B_{i}}\right)=1 \text {. }
$$

Since $Q_{i}$ is a sum of identically distributed Gaussian random variables (the $V_{i j}$ ), each with mean zero, and the sum of the squares of the coefficients (the $A_{i j} / B_{i}$ ) is unity, $Q_{i}$ itself has a Gaussian distribution with variance $M^{2}$ and mean zero. From a set of measured values of $Q$ in a nucleus, one can extract $M^{2}$ as the average value of $Q^{2}$.

In order to determine the value of $M^{2}$ when the spins of the $p$-wave levels are not known or there are experimental errors, the method of likelihood analysis can be used. When neutrons scatter from a spin-zero target of mass $A$, resonances in the $A+1 \mathrm{CN}$ are formed. The $p-1 / 2$ resonances, which mix with nearby $s-1 / 2$ resonances, can have large 
PV longitudinal asymmetries; $p-3 / 2$ resonances cannot. The likelihood function, $L(M)$, must take account of this ambiguity if the spins are not known. The likelihood function, $L(M)$, is given by:

$$
L(M)=\prod_{i=1}^{n} \frac{1}{3} \frac{1}{\sqrt{2 \pi\left(M^{2}+\sigma_{i}^{2}\right)}} \exp \left(\frac{-P_{i}^{2}}{2\left(M^{2}+\sigma_{i}^{2}\right)}\right)+\frac{2}{3} \frac{1}{\sqrt{2 \pi \sigma_{i}^{2}}} \exp \left(\frac{-P_{i}^{2}}{2 \sigma_{i}^{2}}\right) .
$$

If the spins are known, the second terms are absent. Using Eq. (4) to determine $M$ for the ${ }^{238} \mathrm{U}$ data gives $M=0.57_{-0.31}^{+} \mathrm{meV}$. For ${ }^{232} \mathrm{Th}$, the result is $M=1.39+0.55 \mathrm{meV}$. If Eq. (4) is modified to take the non-zero average asymmetry into account, the result is $M=$ $1.20 \pm 8.55 \mathrm{meV}^{14}$ The value of $M$ for ${ }^{232}$ Th does not depend strongly on which assumption is made for the constant asymmetry. The values of $M$ for ${ }^{232} \mathrm{Th}$ and ${ }^{238} \mathrm{U}$ are in rough agreement.

\section{Theory of the Random Asymmetry}

Johnson et al. ${ }^{15}$ developed a relationship between $M^{2}$ and the weak mesonexchange couplings based on the theory of statistical nuclear spectroscopy developed by French, Tomsovi,c and collaborators. ${ }^{16}$ The French-Tomsovic theory, based on the central limit theorem, trace algebra, and group theory, represents the strength function of a symmetry-breaking operator,

$$
\frac{2 \pi M^{2}}{d}=S\left(E_{1}, E_{2}\right)
$$

as a bivariant Gaussian function in the arguments $E_{1}$ and $E_{2}$. French and Tomsovic work in a model space of the $0-\hbar \omega$ excitations available to valence particles outside a doubly-closed core. The Hamiltonian is divided into three parts: an independent particle part, a strong two-body residual interaction (taken as a surface $\delta$ function), and a symmetry-breaking part. The parameters of the strength function are determined as expectations of commutators of the symmetry-breaking interaction with the strong Hamiltonian. The strength of the residual interaction is adjusted to fit observed level densities. Johnson et al..$^{15}$ used the fits of French and Tomsovi ${ }^{16}$ to ${ }^{232} \mathrm{Th}$ and ${ }^{238} \mathrm{U}$ as a starting point.

Johnson $e t$ al. assumed that the ratio of the mean-squared matrix element of the PV interaction and of the traceless surface $\delta$ function symmetry-breaking interaction of French and Tomsovic is independent of the selection of CN states over which the average is taken. This would happen, for example, if the $\mathrm{CN}$ states were all random admixtures of all basis states as in the random-matrix approach. ${ }^{17}$ Johnson et al. took the full two-body meson-exchange potential as the PV symmetry-breaking interaction. ${ }^{18}$ Using the fact that 
the trace of an operator is independent of representation, Johnson et al. were able to simplify the calculation of the strength function of the meson-exchange interaction. The calculations showed that only the isovector $\pi$ coupling, $F_{\pi}$, and the isoscalar $\rho$ coupling, $F_{\rho}$, play a significant role in determining $M^{2}$. The strength function for the mesonexchange interaction is a bilinear form in the couplings $F_{\pi}$ and $F_{\rho}$.

$$
M^{2}=\alpha F_{\pi}^{2}+2 \beta F_{\pi} F_{\rho}+\gamma F_{\rho}^{2}
$$

Johnson et al. found the cross term to be negligible. Figure 3 shows constraints on $F_{\pi}$ and $F_{\rho}$ using measured values of $M^{2}$ (the doughnut-shaped region) and measurements of PV observable in light nuclei (the regions bounded by straight lines). The theory relating the observed asymmetry in ${ }^{18} \mathrm{~F}$ to $F_{\pi}$ and $F_{\rho}$ is constrained by measured quantities such as lifetimes and beta decay rates, and therefore the constraint from the ${ }^{18} \mathrm{~F}$ measurement should be given more credence than the others, which are based on $a b$ initio calculations. Together, the ${ }^{18} \mathrm{~F}$ and $M^{2}$ measurements constrain $F_{\pi} \sim 0.0$ and $F_{\rho} \sim 0.5$. These values of the weak meson-nucleon couplings are of the order of quark-model estimates. ${ }^{19}$ The matrix element between $s$ and $p$ single-particle configurations is then calculated to be $\left\langle s\left|V_{\mathrm{PV}}\right| p\right\rangle \sim 1 \mathrm{eV}$ using these values of the coupling constants. Auerbach ${ }^{20}$ obtained a similar value, $0.2 \mathrm{eV}$, using a doorway approach. This estimate of the single-particle matrix element will be important in the next section.

\section{Theories of the Constant Asymmetry}

On the basis of the data available before the new results reported here, we interpreted the tendency of measured asymmetries in ${ }^{232} \mathrm{Th}$, and perhaps in other nuclei, to mean that there is a non-zero average asymmetry, $\overline{P \sqrt{E}}=36 \pm 12 \% \sqrt{\mathrm{eV}}$. This non-zero average value of asymmetries came as a surprise. Only a brief account of the subject will be given here. Equation (1) expresses the PV asymmetry as a ratio involving three matrix elements: $V_{i j}, g_{j}$, and $g_{i}$. Bowman, Garvey, Gould, Hayes, and Johnson ${ }^{14}$ showed that $V_{i j}$ was correlated to the ratio $g_{i} / g_{j}$ and the energy denominator $E_{i}-E_{j}$. The decay amplitudes are written as:

$$
\begin{aligned}
& g_{j}=g^{p}\langle j \mid G+p\rangle \\
& g_{i}=g^{s}\langle i \mid G+s\rangle,
\end{aligned}
$$

where $g^{s}$ and $g^{p}$ are the decay amplitudes of $s$ and $p$ single particles coupled to the target ground state, $G$. The $C N$ states are correspondingly decomposed:

$$
\begin{aligned}
& |j\rangle=|G+p\rangle\langle G+p \mid j\rangle+\left|j^{\prime}\right\rangle \\
& |i\rangle=|G+s\rangle\langle G+s \mid i\rangle+\left|i^{\prime}\right\rangle .
\end{aligned}
$$




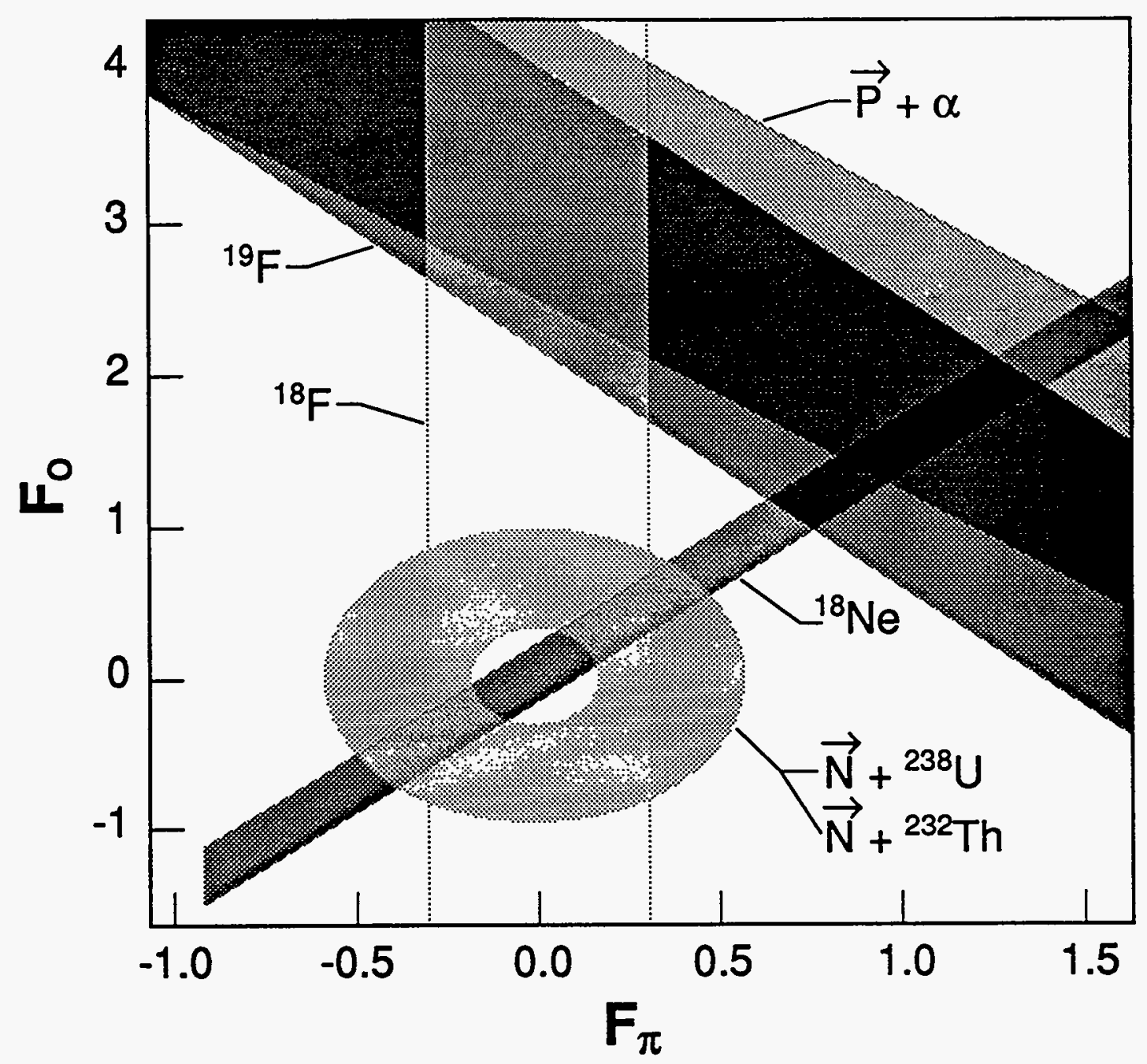

Fig. 3. Constraints on the isovector $\pi, F_{\pi}$, and the isoscalar $\rho, F_{\rho}$, weak meson-nucleon couplings imposed by various experiments. ${ }^{14,18}$

The constant asymmetry, $B$, originates from the single-particle parts of the states and the fluctuating asymmetry from the primed parts.

$$
\begin{aligned}
B \sqrt{\frac{1 \mathrm{eV}}{E_{i}}} & =2 \frac{g^{s}}{g^{p}} \sum_{j} \frac{\langle j \mid G+s\rangle\left\langle G+s\left|V_{\mathrm{PV}}\right| G+p\right\rangle\langle G+p \mid i\rangle}{E_{i}-E_{j}} \frac{\langle G+s \mid j\rangle}{\langle i \mid G+p\rangle} \\
& =2 \frac{g^{s}}{g^{p}} \sum_{j} \frac{\left.\langle j \mid G+s\rangle\right|^{2}\left\langle G+s\left|V_{\mathrm{PV}}\right| G+p\right\rangle}{E_{i}-E_{j}} .
\end{aligned}
$$


The probability $|\langle G+s \mid j\rangle|^{2}$ is concentrated in two peaks located $\hbar \omega$ above and below $E_{i}$. The sum is evaluated by closure yielding:

$$
B \sqrt{\frac{1 \mathrm{eV}}{E_{i}}}=4 \frac{g^{s}}{g^{p}} \frac{\left\langle G+s\left|V_{\mathrm{PV}}\right| G+p\right\rangle}{\hbar \omega}
$$

Using the value of the average asymmetry from section $2, \overline{P \sqrt{E}}=36 \pm 2 \% \sqrt{\mathrm{eV}}$ and $\hbar \omega=7 \mathrm{MeV}$ yields $\left\langle G+s\left|V_{\mathrm{PV}}\right| G+p\right\rangle=500 \mathrm{eV}$. This value of the single-particle matrix element is more than 100 times larger than the values of the single-particle matrix element estimated above from experimental vales of $M, 1.0 \mathrm{eV}$. It is also $100-1000$ times larger than would be estimated from the strength of the weak hadronic interaction, $G_{F} k_{F}^{2}{ }^{19}$ Several other approaches have been used to relate the strength of the weak interaction to the observed size of the constant asymmetry. Koonin, Johnson, and Vogel ${ }^{21}$ use an optical model approach; Lewenkopf and Weidenmüller ${ }^{22}$ use formal reaction theory; and Auerbach and Bowman ${ }^{23}$ use the doorway model. All find the strength of the weak interaction to be 100-1000 times larger than expected.

\section{New Experimental Results}

In 1993 the TRIPLE collaboration carried out experiments on the following targets, ${ }^{238} \mathrm{U},{ }^{232} \mathrm{Th},{ }^{127} \mathrm{I},{ }^{115} \mathrm{In},{ }^{113} \mathrm{Cd}$, and $\mathrm{Ag}(107+109)$. The data are still under analysis, but many new PV asymmetries were observed and the $1991{ }^{232} \mathrm{Th}$ results were confirmed. Figure 4 shows the 1993 asymmetry data for ${ }^{232} \mathrm{Th}$. The statistical errors in these data are more than an order of magnitude smaller than in earlier data due to the increased size of the polarizer aperture $(4 \mathrm{~cm} \rightarrow 8 \mathrm{~cm})$, the increased polarization of the neutron beam $(25 \% \rightarrow 70 \%)$, and the increased detector efficiency.

Table 1 summarizes the results for the signs of PV asymmetries with greater than 3 standard deviation effects. Nuclei other than ${ }^{232}$ Th have random signs, while 8 out of 8 asymmetries in ${ }^{232} \mathrm{Th}$ have positive signs. The probability of 8 of 8 random numbers having a common sign is $2^{-7}=0.8 \%$. The average of the non-zero asymmetries (multiplied by $\sqrt{E}$ to remove the threshold dependence of the PV asymmetry) is $B=36 \pm 14 \%$ $(\mathrm{eV})^{1 / 2}$, where the error was determined from the fluctuations in the data. These two statistics that the probability that the sign consistency in ${ }^{232} \mathrm{Th}$ is unlikely to be a statistical fluke. On the other hand, the results for other nuclei show that the consistency of signs is not a property of all nuclei. This fact rules out all of the theories of the sign consistency phenomenon discussed above. All predict a universal sign consistency. 14,21-23

The most likely explanation of the sign consistency phenomenon seems to be that some narrow local doorway is responsible. The amplitude of a doorway state (such as the configuration $|G+p\rangle$ discussed above in Ref. 14) to occur in a compound nuclear state $|\mu\rangle$ can be estimated using the picket fence model of Bohr Mottelson. ${ }^{24}$ 


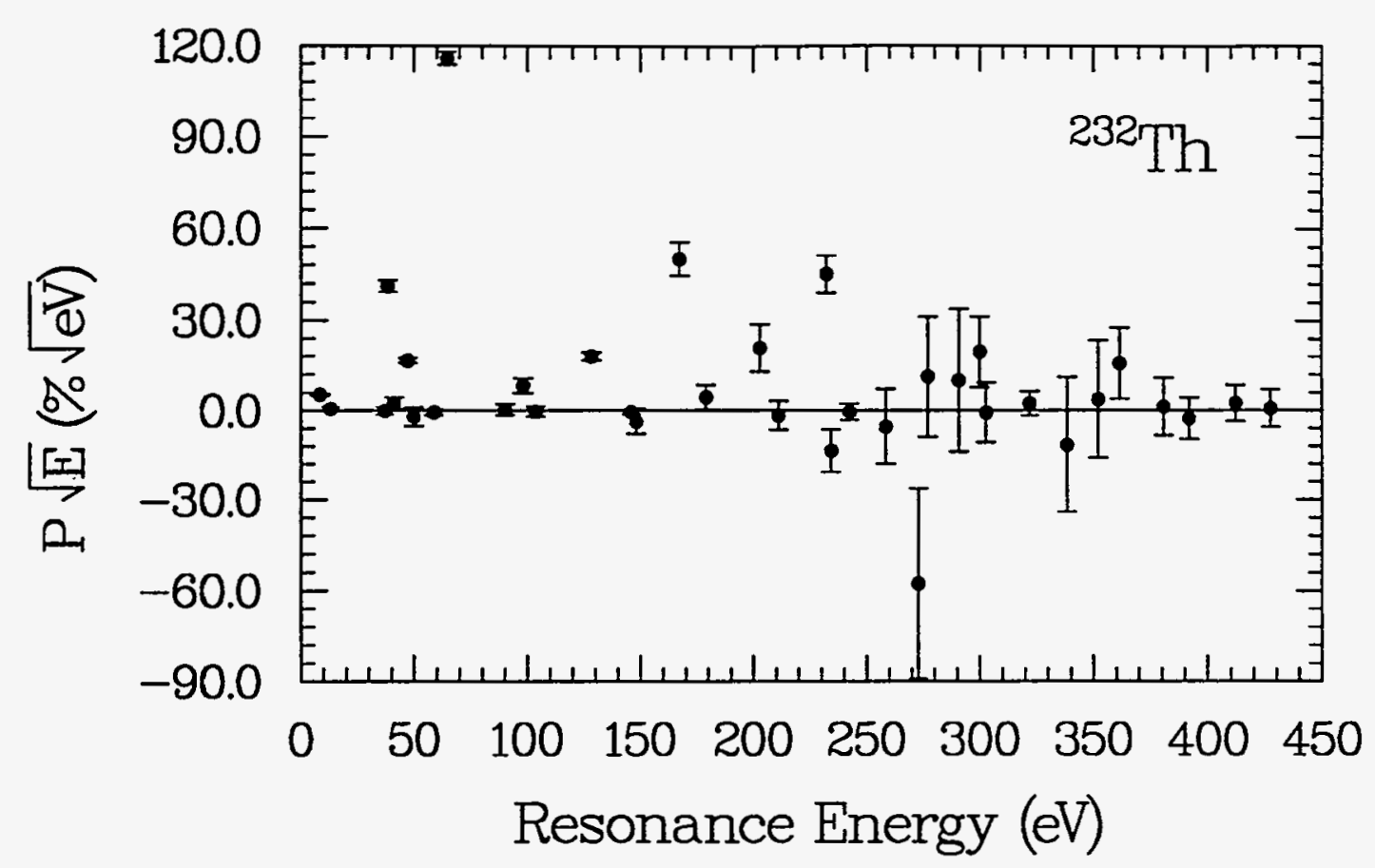

Figure 4. New ${ }^{232}$ Th results. The horizontal axis is the neutron kinetic energy of the compound nuclear resonance and the vertical axis is the parity violating asymmetry of each resonance multiplied by $\sqrt{E}$.

Table 1. The numbers of asymmetries with greater than 3 standard deviation statistical significance with positive and negative signs for different targets.

\begin{tabular}{lcc}
\hline Target & $P>0$ & $P<0$ \\
${ }^{238} \mathrm{U}$ & 3 & 2 \\
${ }^{232} \mathrm{Th}$ & 8 & 0 \\
${ }^{127} \mathrm{I}$ & 0 & 1 \\
${ }^{115} \mathrm{In}$ & 2 & 1 \\
${ }^{113} \mathrm{Cd}$ & 2 & 1 \\
${ }^{107} \mathrm{Ag} /{ }^{109} \mathrm{Ag}$ & 5 & 4 \\
\hline & $|\langle D \mid \mu\rangle|^{2}=\frac{\Gamma d}{2 \pi\left[\left(E_{D}-E_{\mu}\right)^{2}+\Gamma^{2} / 4\right]} \cdot$
\end{tabular}

The maximum of the amplitude squared occurs at $E_{D}=E_{\mu}$. The doorway states in the models of section 5 all assumed that the doorway states involved had spreading widths $G$ of a few $\mathrm{MeV}$ and level spacings of tens of $\mathrm{eV}$, which leads to: 


$$
|\langle D \mid \mu\rangle|^{2}=\frac{2 d}{\pi \Gamma} \approx 10^{-5} .
$$

The small values of the amplitudes of the doorway states in the compound nuclear wave functions led to the small values of the average asymmetry. If the spreading width of the doorway is assumed to be narrow compared to a few $\mathrm{MeV}$, large values of the average asymmetry are possible. If the width of the doorway is small, then it can be admixed into a small number of compound nuclear states with large amplitudes and produce sign correlations for those states. The lack of a universal sign effect would then be explained by the need for the accidental occurrence of the appropriate doorway near neutron threshold. A challenge is to formulate a model of the nuclear structure of this hypothetical doorway that relates its weak matrix elements to its neutron decay amplitude, and suggests experiments that test the theory of its structure.

The 1993 data are under intense analysis. They will be used to extract values of the mean squared weak matrix elements of the parity interaction between compound nuclear states, $M^{2}$. These values of $M^{2}$ can in turn be used to constrain the weak mesonnucleon couplings $F_{\pi}$ and $F_{\rho}$. The consistency of the constraints developed for the meson-nucleon couplings using data from different regions of nuclear mass will serve to test the validity of the theories that relate $M^{2}$.

\section{Acknowledgments}

This work was supported in part by the U.S. Department of Energy, Office of High Energy and Nuclear Physics, under grants No. DE-FG05-88-ER40441 and No. DE-FG05-91-ER40619 and by the U.S. Department of Energy, Office of Energy Research, under Contract No. W-7405-ENG-36.

\section{References}

1. N. R. Roberson, C. D. Bowman, J. D. Bowman, P. P. Delheij, C. M. Frankle, C. R. Gould, D. G. Haase, J. N. Knudson, G. E. Mitchell, R. N. Mortensen, S. Penttilä, H. Postma, S. J. Seestrom, J. J. Szymanski, S. A. Wender, S. H. Yoo, V. W. Yuan, and X. Zhu, Nucl. Instrum. Methods A 326, 549 (1993).

2. S. I. Penttilä, J. D. Bowman, P. P. J. Delheij, C. M. Frankle, D. G. Haase, R. Mortensen, H. Postma, S. J. Seestrom, and Yi-Fen Yen, in Time Reversal Invariance and Parity Violation in Neutron Reactions, C. R. Gould, J. D. Bowman, and Yu. Popov, eds. (World Scientific, Singapore, 1994).

3. Y.-F. Yen, J. D. Bowman, Y. Matsuda, S. I. Penttilä, H. M. Shimizu, R. W. Strickland, S. Takahashi, S. H. Yoo, and V. W. Yuan, in Time Reversal Invariance and Parity Violation in Neutron Reactions, C. R. Gould, J. D. Bowman, and Yu. Popov, eds. (World Scientific, Singapore, 1994).

4. V. P. Alfimenkov et al., Nucl. Phys. A 398, 93 (1983). 
5. J. R. Vanhoy et al., Z. Phys. A 333, 229 (1988).

6. V. E. Bunakov and V. P. Gudkov, Nucl. Phys. A 401, 93 (1983).

7. S. A. Biryukov, L. N. Bondarenko, S. V. Zhukov, Yu. V. Zakharov, V. M. Zykov, V. L. Kuznetsov, P. V. Malankin, V. I. Mostovoi, A. A. Osochnikov, S. P. Pugachev, V. I. Raitsis, and A. N. Chernvi, Yad. Fiz. 45, 1511 (1987).

8. Y. Masuda, T. Adachi, A. Masaike, and K Morimoto, Nucl. Phys. A 504, 269 (1989).

9. V. W. Yuan, C. D. Bowman, J. D. Bowman, J. E. Bush, P. P. J. Delheij, C. M. Frankle, C. R. Gould, D. G. Haase, J. N. Knudson, G. E. Mitchell, S. Penttilä, H. Postma, N. R. Roberson, S. J. Seestrom, J. J. Szymanski, and X. Zhu, Phys. Rev. C 44, 2187 (1991).

10. J. D. Bowman, C. D. Bowman, J. E. Bush, P. P. Delheij, C. M. Frankle, C. R. Gould, D. G. Haase, J. N. Knudson, G. E. Mitchell, S. Penttilä, H. Postma, N. R. Roberson, S. J. Seestrom, J. J. Szymanski, V. W. Yuan, and X. Zhu, Phys. Rev. Lett. 65, 1192 (1990).

11. C. M. Frankle, J. D. Bowman, J. E. Bush, P. P. Delheij, C. R. Gould, D. G. Haase, J. N. Knudson, G. E. Mitchell, S. Penttilä, H. Postma, N. R. Roberson, S. J. Seestrom, J. J. Szymanski, S. H. Yoo, V. W. Yuan, and X. Zhu, Phys. Rev. Lett. 67, 564 (1991).

12. V. P. Alfimenkov, Yu. D. Mareev, L. B. Pikelner, V. R. Skoy, and V. N. Shvetsov, Yad. Fiz. 54, 1489 (1991).

13. V. P. Alfimenkov, S. B. Borzakov, Vo Van Thuan, Yu. D. Mareev, L. B. Pikelner, A. S. Khrykin, and E. I. Sharapov, Nucl. Phys. A 398, 93 (1983).

14. J. D. Bowman, G. T. Garvey, C. R. Gould, A. C. Hayes, and M. B. Johnson. Phys. Rev. Lett. 68, 780 (1992).

15. M. B. Johnson, J. D. Bowman, and S. H. Yoo, Phys. Rev. Lett. 67, 310 (1991).

16. J. B. French, V. K. B. Kott, A. Pandy, and S. Tomsovic, Ann. Phys. (N.Y.) 181, 260 (1988).

17. V. E. Bunakov, E. D. Davis, and H. A. Weidenmüller, Phys. Rev. C 42, 1718 (1990).

18. E. G. Adelberger and W. C. Haxton, Annu. Rev. Nucl. Part. Sci. 35, 501 (1985).

19. B. Deplanques, J. F. Donoghue, and B. R. Holstein, Ann. Phys. (N.Y.) 124, 449 (1980).

20. N. Auerbach, Phys. Rev. C 45, R514 (1992).

21. S. E. Koonin, C. W. Johnson, and P. Vogel, Phys. Rev. Lett. 69, 1163 (1992).

22. C. H. Lewenkopf and H. A. Weidenmüller, Phys. Rev. C 46, 2601 (1992).

23. N. Auerbach and J. D. Bowman, Phys. Rev. C 46, 2582 (1992).

24. A. Bohr and B. Mottelson, in Nuclear Structure, (Benjamin, New York, 1969), Vol. 1. 